\title{
The Relationship between Music Property and Degree of its Popularity in Spotify
}

\author{
Han Chen \\ Southeast University, Nanjing, 210000, China \\ chan990907@gmail.com
}

\begin{abstract}
The global recorded music market grew by $7.4 \%$ in 2020 , the sixth consecutive year of growth, according to IFPI, the organization that represents the recorded music industry worldwide. Figures released today in IFPI's Global Music Report show total revenues for 2020 were US\$21.6 billion. Growth was driven by streaming, especially by paid subscription streaming revenues, which increased by $18.5 \%$. There were 443 million users of paid subscription accounts at the end of 2020 . Total streaming (including both paid subscription and advertising-supported) grew $19.9 \%$ and reached $\$ 13.4$ billion, or $62.1 \%$ of total global recorded music revenues. The growth in streaming revenues more than offset the decline in other formats' revenues, including physical revenues which declined 4.7\%; and revenues from performance rights which declined $10.1 \%$ - largely as a result of the COVID-19 pandemic. As we have seen, the music market has a huge economic potential on a global scale, then I want to use 40,000 of data in Spotify to analysis people's average hobbies and build a simple persona.
\end{abstract}

Keywords: Liveness; Danceability; Property; Popularity.

\section{Introduction}

Music is the art of arranging sounds in time to produce a composition through the elements of melody, harmony, rhythm, and timbre.[1] [2] It is one of the universal cultural aspects of all human societies. General definitions of music include common elements such as pitch (which governs melody and harmony), rhythm (and its associated concepts tempo, meter, and articulation), dynamics (loudness and softness), and the sonic qualities of timbre and texture (which are sometimes termed the "color" of a musical sound). Different styles or types of music may emphasize, de-emphasize or omit some of these elements. Music has lots of properties such as the DURATION, DYNAMICS and Melody, DURATION can refer to the length of individual notes or even whole songs or Beat and pulse even the Rhythmic patterns. DYNAMICS is referred to the volume of individual notes or even whole songs and some changes in volume or sudden (block dynamics) and gradual (crescendo, decrescendo). MELODY is all about the horizontal arrangement of sound. I can refer to: A sequence of single notes (sung or played) or the contour of the melody or patterns such as phrases, riffs, sequences, motifs. By contrast, it hard to say that everyone listening to music with so many rules, they choose the music that they like. There is no special definition of "like", maybe they just choose the type of music style they love or the song they can dance with, in this article, we will use energy, liveness and danceability to analysis.

\section{The Elements of Music in Spotify}

Analyze the nature of a whole song from three dimensions, first its energy the energy is the power that a song has, so it is a index of the power of the song, the higher the energy is the more power it has. The second part is the liveness, it the target used to identify whether the song is a live song. Many people prefer to live songs than some studio song, it depends on own habits, then we can find out how many live songs in this data using liveness. If the liveness higher than 0.80 then we can consider it as a live song. The last part is the danceability, dance is connected with music and can not leave without music, the danceability means the index of the possibility to dance with this music, the higher the value, the more likely to dance with the song. 


\subsection{Part 1}

When we first look at the dataset, it has 42305 records about the music in the dataset, there has the index of the danceability, energy, acousticness, liveness and so on.

\begin{tabular}{|c|c|c|c|c|c|c|c|c|c|c|c|c|c|}
\hline & danceability & energy & key & loudness & mode & speechiness & acousticness & instrumentalness & 1iveness & valence & tempo & type & \\
\hline 0 & 0.831 & 0.814 & 2 & -7.364 & 1 & 0.4200 & 0.059800 & 0.013400 & 0.0556 & 0.3890 & 156.985 & audio_features & $2 \mathrm{Vc}$ \\
\hline 1 & 0.719 & 0.493 & 8 & -7.230 & 1 & 0.0794 & 0.401000 & 0.000000 & 0.1180 & 0.1240 & 115.080 & audio_features & $7 p c$ \\
\hline 2 & 0.850 & 0.893 & 5 & -4.783 & 1 & 0.0623 & 0.013800 & 0.000004 & 0.3720 & 0.0391 & 218.050 & audio_features & OvSv \\
\hline 3 & 0.476 & 0.781 & 0 & -4.710 & 1 & 0.1030 & 0.023700 & 0.000000 & 0.1140 & 0.1750 & 186.948 & audio_features & OVs \\
\hline 4 & 0.798 & 0.624 & 2 & -7.668 & 1 & 0.2930 & 0.217000 & 0.000000 & 0.1660 & 0.5910 & 147.988 & audio_features & $4 \mathrm{j} \mathrm{C}_{\mathrm{f}}$ \\
\hline ... & $\ldots$ & $\ldots$ & $\ldots$ & $\ldots$ & $\ldots$ & $\ldots$ & $\ldots$ & $\ldots$ & $\ldots$ & ... & $\ldots$ & $\ldots$ & \\
\hline 42300 & 0.528 & 0.693 & 4 & -5.148 & 1 & 0.0304 & 0.031500 & 0.000345 & 0.1210 & 0.3940 & 150.013 & audio_features & $4 i$ \\
\hline 42301 & 0.517 & 0.768 & 0 & -7.922 & 0 & 0.0479 & 0.022500 & 0.000018 & 0.2050 & 0.3830 & 149.928 & audio_features & Oht \\
\hline 42302 & 0.361 & 0.821 & 8 & -3.102 & 1 & 0.0505 & 0.026000 & 0.000242 & 0.3850 & 0.1240 & 154.935 & audio_features & 72 \\
\hline 42303 & 0.477 & 0.921 & 6 & -4.777 & 0 & 0.0392 & 0.000551 & 0.029600 & 0.0575 & 0.4880 & 150.042 & audio_features & $6 t$ \\
\hline 42304 & 0.529 & 0.945 & 9 & -5.862 & 1 & 0.0615 & 0.001890 & 0.000055 & 0.4140 & 0.1340 & 155.047 & audio_features & $6 \mathrm{M}$ \\
\hline
\end{tabular}

Fig 1. Dataset

Then we use the function info () to check if there has any missing data, luckily, there has not missing anything. So we can use this dataset to do some deeper analysis.

\subsection{Part 2}

Music can be described in terms of many genres and styles. Classifications are often arbitrary, and may be disputed and closely related forms often overlap. Larger genres and styles comprise more specific sub-categories. In this part we will find out which style is the most popular style of this whole list. The more this kind of style's music they listening, the more they love this style. We can use function unique () to find out how many genres we have in this list.

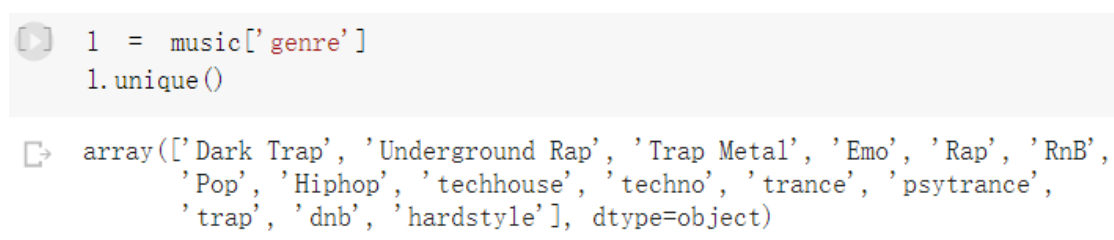

\section{Fig 2. Function}

After we know how many kinds of styles we have, we can use these to build a new column to calculate the counts of each style to find out the most famous kind and draw a picture about it.

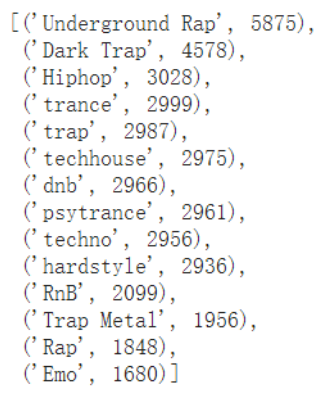

Fig 3. Build a new column to calculate the counts of each style 
Volume 13 (2021)

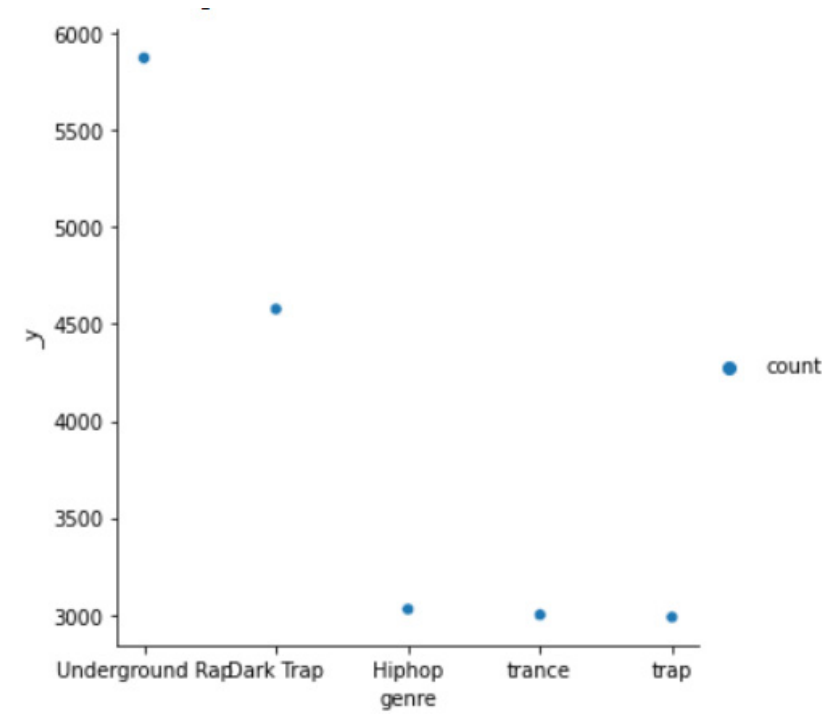

Fig 4. Draw a picture

\subsection{Part 3}

The most attractive parts of music is its melody and the good or bad of melody can be measured by the danceability and valence. When we want to create a song and already set down the approximately value of the danceability then what value of the valence is better for us to choose? If we can figure out this problem, we can be more successful. Valence is a measure from 0.0 to 1.0 describing the musical positiveness conveyed by a track. Tracks with high valence sound more positive (e.g. happy, cheerful, euphoric), while tracks with low valence sound more negative (e.g. sad, depressed, angry). Then we use plt.figure to create a picture of the two values, and the function regr.predict to predict the better value of danceability and valence. The red line is predicted line.

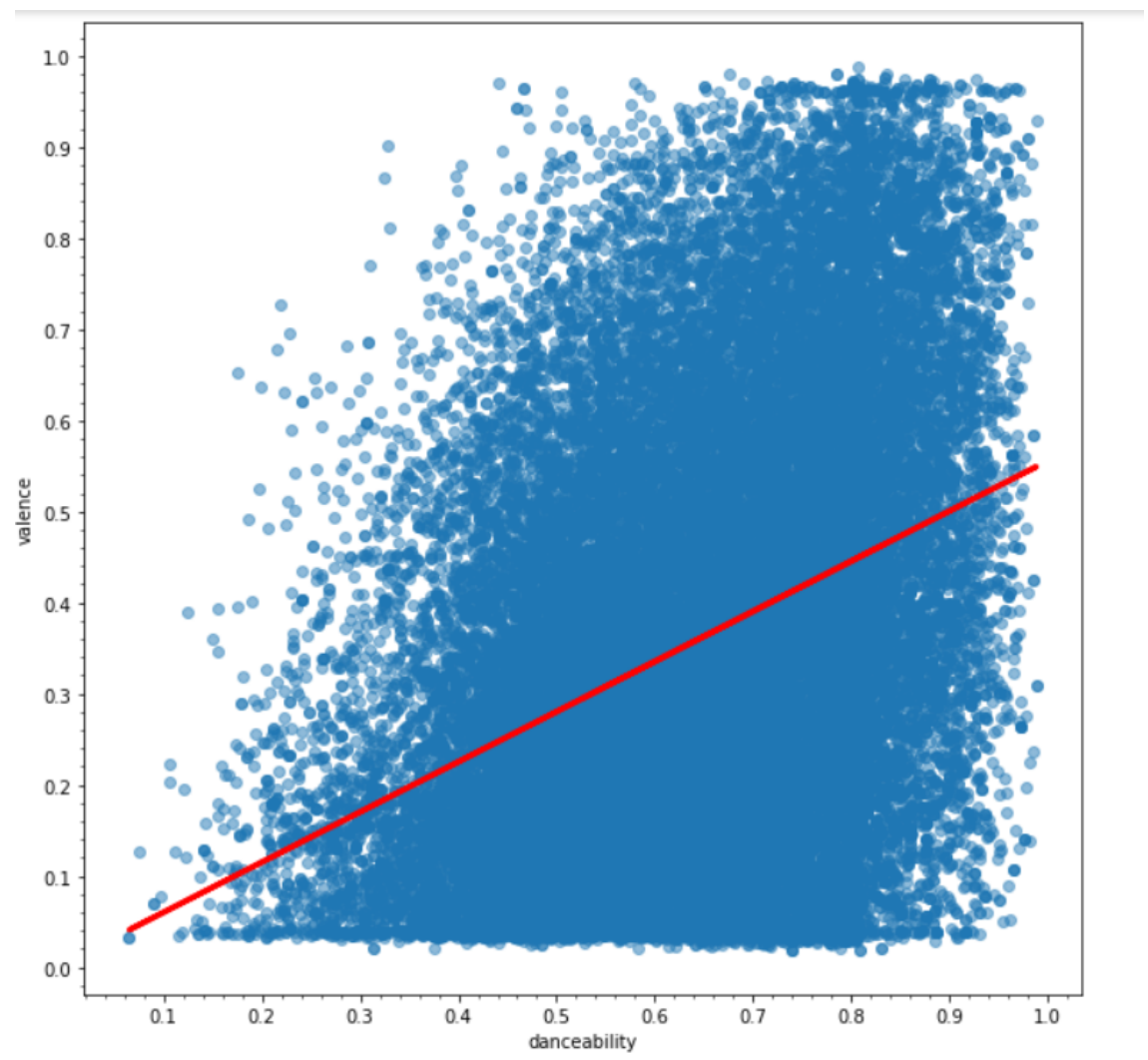

Fig 5. Danceability 


\subsection{Part 4}

There have three main values of a song: energy, danceability and the valence. Energy is a term that a lot of musicians use but find hard to define. You might use the word energy in a very nebulous sort of way, where it's synonymous with intensity. With that usage of the word, most listeners of music would know what you mean if you said, "I really like the energy of this song." They would take that to mean that the music is probably loud, rhythmically active, and is the kind of music that gets the blood pumping.

If you're a songwriter, you can use a more sophisticated concept of energy to ensure that you captivate listeners, and keep them listening right to the end of your song. Then we can find a relationship between the energy we have and danceability to create a popular song.
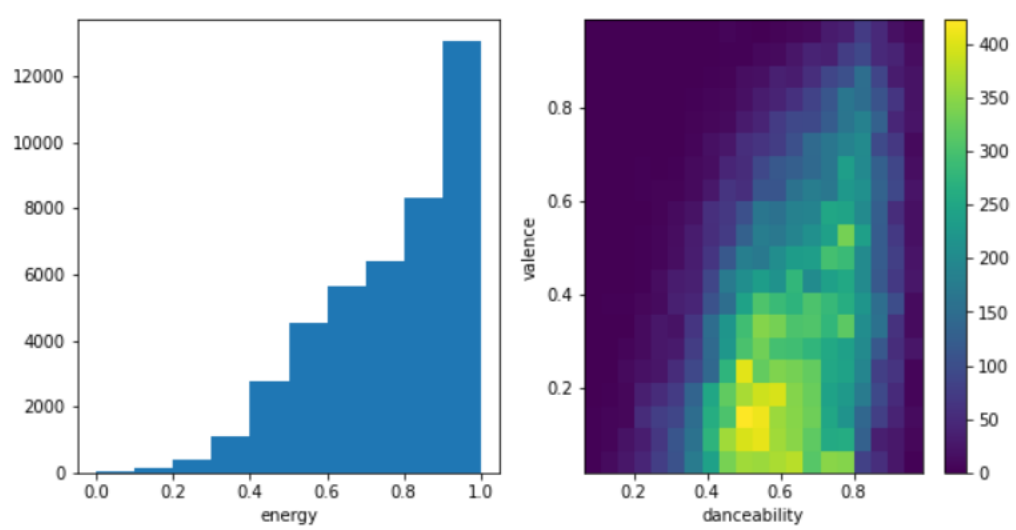

Fig 6. Relationship between the energy we have and danceability to create a popular song

\subsection{Part 5}

The last part is the liveness of a song. Live music is a kind of performance like the concert, A performance in front of audiences. The performance may be by a single musician, sometimes then called a recital, or by a musical ensemble, such as an orchestra, choir, or band. They are held in a wide variety and size of settings, from private houses and small nightclubs, dedicated concert halls and parks, to large multipurpose buildings, such as arenas and stadiums. The ratio between the danceability and liveness can help us use the best value of danceability when we try to play a live song. First, we use the detail selection to build a new list contain all the songs' liveness higher than 0.8 then compare their danceability to create a new picture.

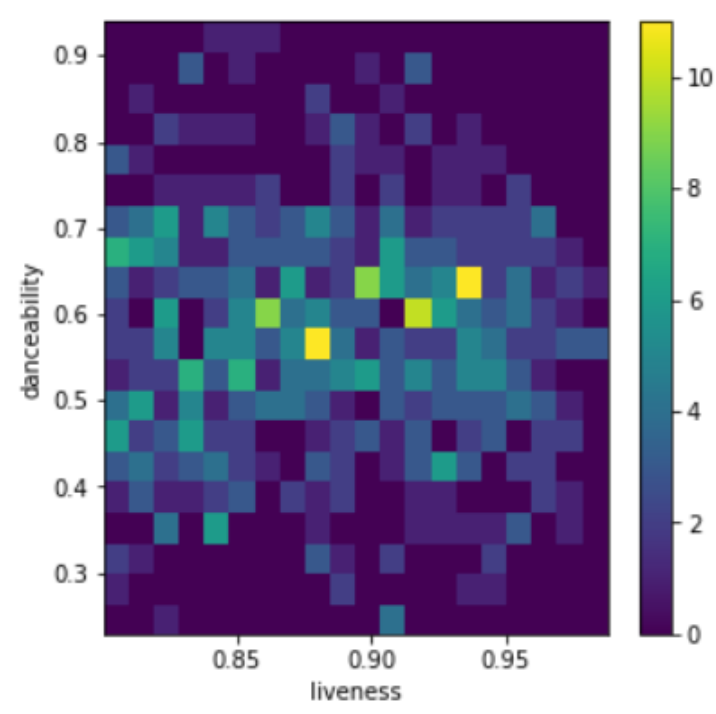

Fig 7. Liveness 
Volume 13 (2021)

\section{References}

[1] Antti Mikael Rousi,Reijo Savolainen,Maaria Harviainen,Pertti Vakkari.Situational relevance of music information modes[J].Journal of Documentation . 2018 (5).

[2] Antti Mikael Rousi, Reijo Savolainen, Pertti Vakkari. A typology of music information for studies on information seeking[J]. Journal of Documentation. 2016 (2).

[3] Zihan Xu. Comparison among Three Popular Music Appraisal Mechanisms in America and Their Contemporary Significance [J].Hundred Schools In Arts,2013, (6):203-206. 